\title{
FUEL EFFICIENT STRATEGIES FOR REDUCING CONTRAIL FORMATIONS IN UNITED STATES AIRSPACE
}

\author{
Banavar Sridhar and Neil Y. Chen, NASA Ames Research Center, Moffett Field, CA \\ Hok K. Ng, University of California, Santa Cruz, Moffett Field, CA
}

\begin{abstract}
This paper describes a class of strategies for reducing persistent contrail formation in the United States airspace. The primary objective is to minimize potential contrail formation regions by altering the aircraft's cruising altitude in a fuel-efficient way. The results show that the contrail formations can be reduced significantly without extra fuel consumption and without adversely affecting congestion in the airspace. The contrail formations can be further reduced by using extra fuel. For the day tested, the maximal reduction strategy has a 53\% contrail reduction rate. The most fuel-efficient strategy has an $8 \%$ reduction rate with $2.86 \%$ less fuel-burnt compared to the maximal reduction strategy. Using a cost function which penalizes extra fuel consumed while maximizing the amount of contrail reduction provides a flexible way to trade off between contrail reduction and fuel consumption. It can achieve a 35\% contrail reduction rate with only $0.23 \%$ extra fuel consumption. The proposed fuel-efficient contrail reduction strategy provides a solution to reduce aviation-induced environmental impact on a daily basis.
\end{abstract}

\section{Introduction}

Aircraft-induced environmental impact has drawn more attention in recent years [1]. The three largest emission impacts include direct emission of greenhouse gases such as $\mathrm{CO}_{2}$, emissions of $\mathrm{NOx}$, and persistent contrails. The emission of $\mathrm{CO}_{2}$ and NOx are related to fuel burn.

Contrails are clouds that are visible trails of water vapor made by the exhaust of aircraft engines. They appear and persist if the aircraft is flying in certain atmospheric conditions. Persistent contrails reduce incoming solar radiation and outgoing thermal radiation in a way that accumulates heat [2]. The global mean contrail cover in 1992 was estimated to double by 2015 , and quadruple by 2050 due to the increase in air traffic [3]. Studies suggest that the environmental impact from persistent contrails is estimated to be three to four times [4], or even ten times [5] larger than aviation-induced emissions. Therefore, concepts to reduce aircraft induced persistent contrail in a fuel efficient way needs to be explored to minimize the impact on the global environment.

Efforts have been made in the past years to reduce the persistent contrail formation. Gierens [6] and Noppel [7] reviewed various strategies for contrail avoidance. Mannstein [8] proposed a strategy to reduce the climate impact of contrails significantly by making small changes in individual flight altitude. Campbell [9] presented a methodology to optimally reroute aircraft trajectories to avoid the formation of persistent contrails with the use of mixed integer programming. Both methodologies require a flexible free flight and onboard contrail detection system. Fichter [10] showed that the global annual mean contrail coverage was reduced by downshifting the cruise altitude. Williams [11], [12] proposed strategies for contrail reduction by identifying fixed and varying maximum altitude restriction policy. These restrictions generally imply more fuel burn, and add congestion in the already crowded airspace at lower altitudes.

The objective of this paper is to develop strategies to reduce persistent contrail formation with consideration of fuel consumption and airspace congestion. Contrail frequency index is used to quantify the severity of contrail formation. The strategy for reducing persistent contrail formations is to minimize contrail frequency index by altering the aircraft's cruising altitude in a fuel-efficient way. A strategy using a cost function which penalizes extra fuel consumed while maximizing the amount of contrail reduction is proposed. It provides a flexible way to trade off between contrail reduction and fuel consumption. The analysis results show that the contrail frequency can be reduced to some extent without extra fuel consumption and without adding congestion to the airspace. The contrail frequency can be further reduced by using extra fuel. 
The remainder of the paper is organized as follows. The Data and Model section provides the descriptions of atmospheric data, contrail model, aircraft data, and contrail frequency index. Next, contrail reduction strategies are described in the Contrail Reduction Strategies section. The results are demonstrated in Results section. Finally, a summary and conclusions are presented in Conclusions section.

\section{Data and Model}

\section{Atmospheric Data}

Contrails can be observed from surface data [13] and detected by satellite data [14]. Duda [15] has related the observations to numerical weather analysis output and showed that persistent contrail formation can be computed using atmospheric temperature and humidity data retrieved from the Rapid Updated Cycle (RUC) data provided by the National Oceanic and Atmospheric Administration (NOAA). Contrails can persist when ambient air is supersaturated with respect to ice, which means that relative humidity with respect to ice (RHi) is greater than one hundred percent [16]. The RHi can be computed from relative humidity with respect to water (RHw) and temperature, which are available in the RUC data. The one-hour forecast and the 40-km RUC data are used in this paper. The data have temporal resolution of one hour, horizontal resolution of forty kilometer, and isobaric pressure level from 100 to 1000 hectopascal $(\mathrm{hPa})$ in $25 \mathrm{hPa}$ increment. The vertical range of interest in this study is from 150 $\mathrm{hPa}$ to $400 \mathrm{hPa}$, which is equivalent to pressure altitude of about 23,600 feet to 44,400 feet. The temperature and $\mathrm{RHw}$ contours at $8 \mathrm{AM}$ eastern daylight time (EDT) on August 1, 2007 at pressure altitude $250 \mathrm{hPa}$, or 34,057 feet, are shown in Fig. 1a and $1 \mathrm{~b}$.

\section{Contrail Model}

Contrails are clouds produced by aircraft at high altitude. The potential persistent contrail formation areas (contrail areas) are defined as areas with RHi greater than or equal to $100 \%$. RHi can be computed from $\mathrm{RHw}$ and temperature using the saturation vapor pressure coefficients of Alduchov [17], formulated as

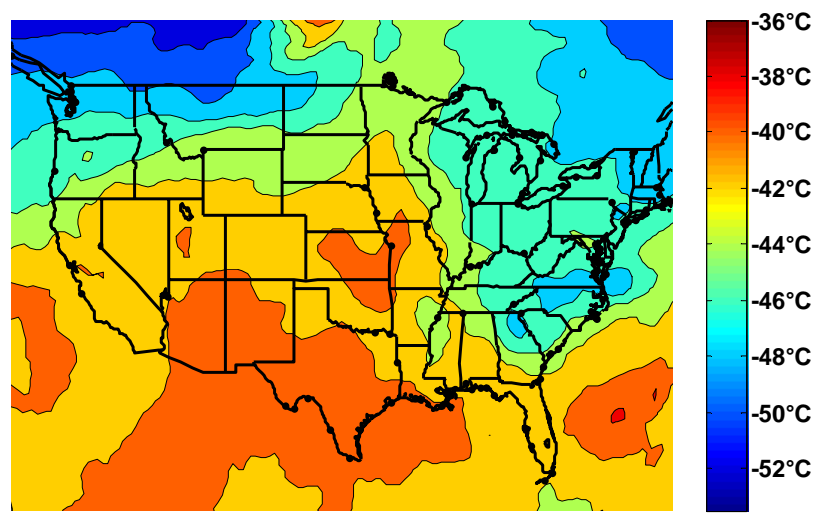

(a) Temperature

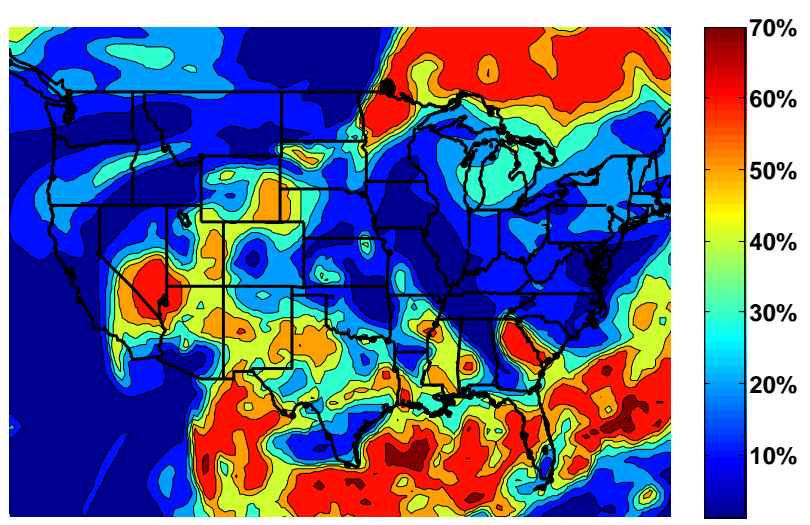

(b) Relative humidity with respect to water

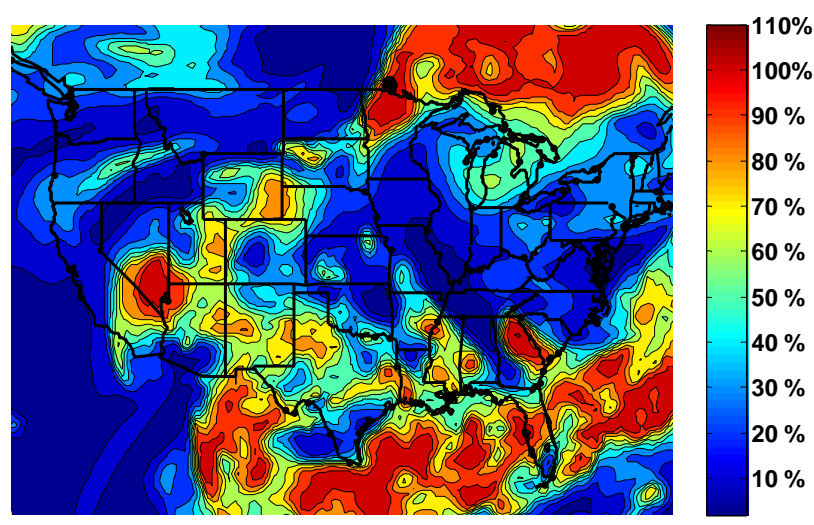

(c) Relative humidity with respect to ice

Figure 1. Contours of temperature, RHw, and RHi at 34,057 feet at 8AM EDT on August 1, 2007.

$$
R H i=R H w \times \frac{6.0612 e^{18.102 T /(249.52+T)}}{6.1162 e^{22.577 T /(237.78+T)}},
$$


where $T$ is the temperature in Celsius. The atmospheric profile shown in Fig. 1a and Fig. 1b can be translated to RHi, as shown in Fig. 1c.

The 40-km RUC data have $(113 \times 151)$ data points. The altitude level index $l$ is defined as $l=1 \ldots 11$ corresponding to isobaric pressure level at $400,375, \ldots, 150 \mathrm{hPa}$. The altitude level, isobaric pressure level, and approximate corresponding flight level are listed in Table 1.

Table 1. Altitude level index, isobaric pressure level, and pressure altitude.

\begin{tabular}{|c|c|c|c|c|c|c|}
\hline Level index & 1 & 2 & 3 & 4 & 5 & 6 \\
\hline $\begin{array}{c}\text { Pressure } \\
\text { level (hPa) }\end{array}$ & 400 & 375 & 350 & 325 & 300 & 275 \\
\hline $\begin{array}{c}\text { Flight level } \\
\text { (100 feet) }\end{array}$ & 236 & 251 & 267 & 283 & 301 & 320 \\
\hline Level index & 7 & 8 & 9 & 10 & 11 & \\
\hline $\begin{array}{c}\text { Pressure } \\
\text { level (hPa) }\end{array}$ & 250 & 225 & 200 & 175 & 150 & \\
\hline $\begin{array}{c}\text { Flight level } \\
\text { (100 feet) }\end{array}$ & 341 & 363 & 387 & 414 & 444 & \\
\hline
\end{tabular}

The potential persistent contrail formation matrix (contrail matrix) at hour $h$ at level $l$ is defined as

$$
\mathbf{R}_{h}^{l}=\left[\begin{array}{cccc}
r_{1,1} & r_{1,2} & \cdots & r_{1,151} \\
\vdots & \vdots & \ddots & \vdots \\
r_{113,1} & r_{113,2} & \cdots & r_{113,151}
\end{array}\right],
$$

where $r_{i, j}$ is 1 if $\mathrm{RHi} \geq 100 \%$ at grid $(\mathrm{i}, \mathrm{j})$, and 0 if $\mathrm{RHi}$ $<100 \%$.

\section{Aircraft Data}

The aircraft data used in this paper are extracted from the aircraft locations provided by the Federal Aviation Administration's (FAA's) Aircraft Situation Display to Industry (ASDI) data. The ASDI has a sampling rate of one minute. The same geometry grid used in the RUC data is used to generate the aircraft position matrix. The aircraft position matrix is defined as

$$
\mathbf{A}_{h}^{l}=\left[\begin{array}{cccc}
a_{1,1} & a_{1,2} & \cdots & a_{1,151} \\
\vdots & \vdots & \ddots & \vdots \\
a_{113,1} & a_{113,2} & \cdots & a_{113,151}
\end{array}\right],
$$

where $a_{i, j}$ is the number of aircraft within grid $(\mathrm{i}, \mathrm{j})$ flying closest to altitude level $l$ at time $t$.

\section{Contrail Frequency Index}

Contrail frequency index is defined as the number of aircraft that would fly through potential contrail formation regions during a period of time. Center contrail frequency index is used to indicate the contrail severity in a given center [18]. To specify the location of the twenty U.S. centers in the grid scale, the center grid matrix is defined as

$$
\mathbf{C}_{\text {center }}=\left[\begin{array}{cccc}
c_{1,1} & c_{1,2} & \cdots & c_{1,151} \\
\vdots & \vdots & \ddots & \vdots \\
c_{113,1} & c_{113,2} & \cdots & c_{113,151}
\end{array}\right],
$$

where $c_{i, j}$ is one if the grid point is within the center and zero if not.

The center contrail frequency index is defined as the number of aircraft flying through contrail area at time $t$ at level $l$, formulated as

$$
\begin{aligned}
C F I_{\text {center }, l, t} & =\operatorname{sum}\left(\mathbf{R}_{t}^{l} \circ \mathbf{A}_{t}^{l} \circ C_{\text {center }}\right) \\
& =\sum_{i=1}^{113} \sum_{j=1}^{151} r_{i, j} a_{i, j} c_{i, j},
\end{aligned}
$$

where $\operatorname{sum}(\bullet)$ is an operator that computes the sum of elements in a matrix, and $\circ$ is the Hadamard product that performs the entrywise multiplication of two matrices. For strategic planning, the predicted contrail frequency index was used for contrail reduction strategies [18]. The predicted index is defined as

$$
P C F I_{\text {center }, i, t}=\operatorname{sum}\left(\hat{\mathbf{R}}_{t}^{l} \circ \hat{\mathbf{A}}_{t}^{l} \circ C_{\text {center }}\right),
$$

where $\hat{\mathbf{R}}_{t}^{l}$ is the contrail matrix computed from forecast weather and $\hat{\mathbf{A}}_{t}^{l}$ is computed from predicted aircraft locations.

\section{Contrail Reduction Strategies}

Contrail reduction strategies using contrail frequency index and fuel-efficient strategies are discussed in this section. 


\section{Use of contrail frequency index}

Contrail frequency indices are used to quantify the severity of contrail formation. The strategy for reducing the persistent contrail formations is to minimize contrail frequency index by altering the aircraft's cruising altitude. Assume the aircraft at altitude level $l$ at center $k$ are made to fly a different level $l^{\prime}$. The contrail frequency index changes to

$$
C F I_{l, t}^{l^{\prime}}=\operatorname{sum}\left(\mathbf{R}_{t}^{l^{\prime}} \circ \mathbf{A}_{t}^{l} \circ C_{\text {center }}\right) .
$$

To solve the problem, with eleven altitude levels, a contrail frequency index matrix is formed as

$$
\mathbf{C F I}_{\text {center }, t}=\left[\begin{array}{cccc}
C F I_{1, t}^{1} & C F I_{2, t}^{1} & \cdots & C F I_{11, t}^{1} \\
C F I_{1, t}^{2} & C F I_{2, t}^{2} & \cdots & C F I_{11, t}^{2} \\
\vdots & \vdots & \ddots & \vdots \\
C F I_{1, t}^{11} & C F I_{2, t}^{11} & \cdots & C F I_{11, t}^{11}
\end{array}\right]
$$

where the diagonal term $C F I_{l, t}^{l}$ is the contrail frequency index at level $l$ before contrail reduction, and $C F I_{l, t}^{l^{\prime}}$ is the contrail frequency index when guiding aircraft at level $l$ to level $l^{\prime}$. The contrail reduction is

$$
\Delta C F I_{l, t}^{l^{\prime}}=C F I_{l, t}^{l}-C F I_{l, t}^{l^{\prime}} .
$$

Note that when $l^{\prime}>l$, not all aircraft have the ability to fly from level $l$ to level $l^{\prime}$. If altitude level $l^{\prime}$ is higher than an aircraft's maximal flight altitude, it stays at level $l$ and is not counted in $C F I_{l, t}^{l^{\prime}}$. In addition, if an aircraft crosses a sector boundary and causes sector overload, it stays at level $l$ and does not add to $C F I_{l, t}^{l^{\prime}}$.

The strategy is to find the smallest element in each column of $\mathbf{C F I}$ center,$t_{\text {. If the aircraft are limited }}$ to alter $\Delta l$ levels, the solution is the smallest element in $\left[C F I_{l, t}^{l-\Delta l} \ldots C F I_{l, t}^{l} \ldots C F I_{l, t}^{l+\Delta l}\right]^{T}$ in each column.

The solution is denoted as $\left[l_{1}^{\prime} \ldots l_{11}^{\prime}\right]$. Each $l_{i}^{\prime}$ means aircraft at flight level $i$ is flying at level $l_{i}^{\prime}$. If $l_{i}^{\prime}=i$, the aircraft at level $i$ did not alter. For example, [ $\left[\begin{array}{lll}1 & 2 & 3\end{array}\right.$ 4468891011 ] means that the aircraft at level 5 is changing to level 4 , and aircraft at level 7 is changing to 8 .
As an example, the contrail frequency index matrix at Atlanta Center at 8AM EDT on August 1, 2007 is

$\left[\begin{array}{ccccccccccc}\mathbf{0} & 0 & 0 & 0 & 0 & 0 & 0 & 0 & 0 & 0 & 0 \\ 0 & \mathbf{0} & 0 & 0 & 0 & 0 & 0 & 0 & 0 & 0 & 0 \\ 0 & 0 & \mathbf{0} & 0 & 0 & 0 & 0 & 0 & 0 & 0 & 0 \\ 0 & 1 & 0 & \mathbf{0} & 0 & 0 & 2 & 0 & 0 & 1 & 0 \\ 28 & 26 & 31 & 33 & \mathbf{1 0} & 52 & 68 & 105 & 51 & 6 & 7 \\ 57 & 74 & 43 & 25 & 13 & \mathbf{7 6} & 104 & 148 & 71 & 5 & 10 \\ 69 & 29 & 44 & 33 & 28 & 105 & \mathbf{1 2 8} & 209 & 132 & 9 & 14 \\ 16 & 12 & 5 & 8 & 5 & 62 & 47 & \mathbf{3 6} & 22 & 6 & 0 \\ 0 & 2 & 0 & 0 & 5 & 65 & 45 & 35 & \mathbf{1 9} & 6 & 0 \\ 0 & 0 & 0 & 0 & 4 & 71 & 108 & 36 & 19 & \mathbf{6} & 0 \\ 0 & 0 & 0 & 0 & 10 & 74 & 122 & 33 & 19 & 0 & \mathbf{0}\end{array}\right]$

The diagonal elements of the matrix are shown in bold for clarity. If the aircraft are only allowed to move one level up or down $(\Delta l=1)$ the matrix becomes

$\left[\begin{array}{ccccccccccc}\mathbf{0} & 0 & \times & \times & \times & \times & \times & \times & \times & \times & \times \\ 0 & \mathbf{0} & 0 & \times & \times & \times & \times & \times & \times & \times & \times \\ \times & 0 & \mathbf{0} & 0 & \times & \times & \times & \times & \times & \times & \times \\ \times & \times & 0 & \mathbf{0} & 0 & \times & \times & \times & \times & \times & \times \\ \times & \times & \times & 33 & \mathbf{1 0} & 52 & \times & \times & \times & \times & \times \\ \times & \times & \times & \times & 13 & \mathbf{7 6} & 104 & \times & \times & \times & \times \\ \times & \times & \times & \times & \times & 105 & \mathbf{1 2 8} & 209 & \times & \times & \times \\ \times & \times & \times & \times & \times & \times & 47 & \mathbf{3 6} & 22 & \times & \times \\ \times & \times & \times & \times & \times & \times & \times & 35 & \mathbf{1 9} & 6 & \times \\ \times & \times & \times & \times & \times & \times & \times & \times & 19 & \mathbf{6} & 0 \\ \times & \times & \times & \times & \times & \times & \times & \times & \times & 0 & \mathbf{0}\end{array}\right]$

where $\times$ indicates an invalid move. The sum of the diagonals, 275, is the center contrail frequency index before reduction. For flight level 7, flying the aircraft one level higher would reduce the contrail frequency from 128 to 47. Similarly, guiding aircraft from level 5 to 4,6 to 5,8 to 9 , and 10 to 11 would reduce contrail frequency. The solution for this example can be denoted as [ [ $\left.\begin{array}{lllllllllll}1 & 2 & 3 & 4 & 4 & 5 & 8 & 9 & 9 & 11 & 11\end{array}\right]$. The center contrail frequency index after reduction is 153, a $44 \%$ reduction. The simulation results are shown in Fig. 2. The color lines indicate the contrail regions at different altitudes. The color dots indicate the aircraft flying through the contrail areas. Different colors 
indicate different altitudes, from blue at flight level 236, to red at 444 . There are 275 dots in Fig. 2a, which is the center contrail frequency at all flight levels. When the reduction strategy is applied, the aircraft flying at flight level 341 (level 7, light green dots) were flying at one level up. The move eliminated all the light green (level 7) in Fig. 2a and only a few yellow dots (level 8) were added in Fig. $2 b$. Also, notice that the red dots (level 10) are eliminated and the number of light blue dots is reduced. The total dots in Fig. $2 b$ are 153.

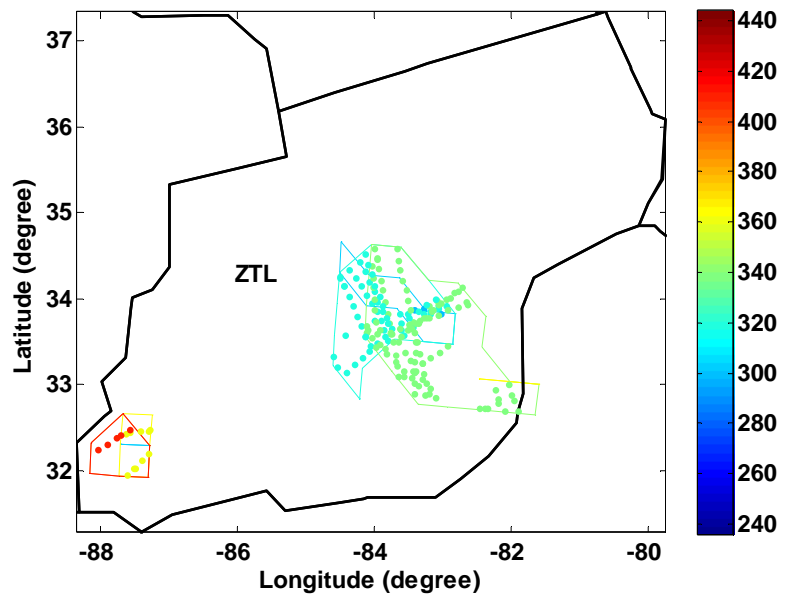

(a) before reduction

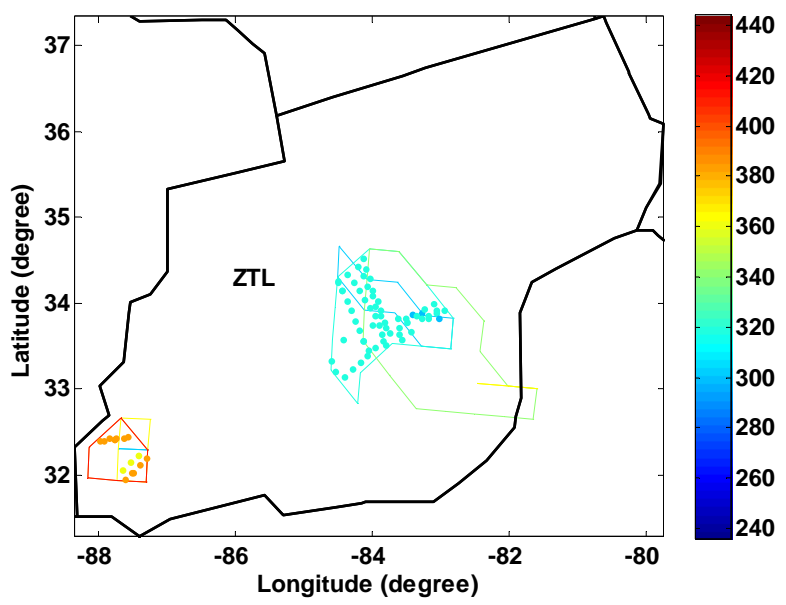

(b) after reduction

Figure 2. Contrail formation area before and after reduction strategy at Atlanta Center at 8AM (EDT) on August 1, 2007.

Note that if the aircraft are allowed to move two levels up or down $(\Delta l=2)$, the contrail frequency can be further reduced to 99 , a $64 \%$ reduction. The more levels the aircraft are allowed to move up or down, the more contrail reduction can be achieved.

\section{Fuel efficient strategies}

Altering cruising altitude changes the aircraft fuel consumption. In order to inspect the environmental impact of contrail reduction strategies, fuel consumption is considered in the strategies. The fuel consumption model used in this analysis is based on the System for assessing Aviation's Global Emissions (SAGE) developed by the FAA [19]. In general, aircraft consume less fuel when cruising at higher altitudes.

The fuel burn matrix is defined as

$$
\mathbf{F B}_{\text {center }, t}=\left[\begin{array}{cccc}
F B_{1, t}^{1} & F B_{2, t}^{1} & \cdots & F B_{11, t}^{1} \\
F B_{1, t}^{2} & F B_{2, t}^{2} & \cdots & F B_{11, t}^{2} \\
\vdots & \vdots & \ddots & \vdots \\
F B_{1, t}^{11} & F B_{2, t}^{11} & \cdots & F B_{11, t}^{11}
\end{array}\right] \text {, }
$$

where the diagonal term $F B_{l, t}^{l}$ is the fuel-burnt for all aircraft at level $l$ at a given center at time $t$ before contrail reduction, and $F B_{l, t}^{l^{\prime}}$ is the total fuel-burnt when guiding aircraft at level $l$ to level $l^{\prime}$. When aircraft are guided from level $l$ to $l^{\prime}$, the extra fuelburnt is

$$
\Delta F B_{l, t}^{l^{\prime}}=F B_{l, t}^{l^{\prime}}-F B_{l, t}^{l} .
$$

$\Delta F B_{l, t}^{l^{\prime}}<0$ implies fuel saving instead of consuming extra fuel. The objective is to achieve a large reduction in contrail frequency index $\left(\Delta C F I_{l, t}^{l^{\prime}}\right)$ with little increase in fuel consumption $\left(\Delta F B_{l, t}^{l^{\prime}}\right)$. In other words, the best move is in the altitude direction with the steepest CFI reduction for unit of extra-fuel consumed.

Consider the same example in the previous subsection. There are four cruising altitude changes. Changing from level 7 to 8,8 to 9 , and 10 to 11 reduces contrail frequency by 88 . These changes reduce fuel-burnt by $1,857 \mathrm{~kg}$. On the other hand, changing from level 5 to 4 and 6 to 5 reduces contrail frequency by 34 with $1,914 \mathrm{~kg}$ of extra fuel. Although the actual environmental cost of contrail frequency is still unknown, it seems unworthy to 
consume $1,914 \mathrm{~kg}$ of fuel to reduce contrail frequency by 34 . The first strategy requires $21 \mathrm{~kg}$ of fuel to reduce a unit of CFI compared to $56 \mathrm{~kg}$ for the second strategy.

Assume the aircraft can move up or down two levels. The CFI matrix is

$$
\left[\begin{array}{ccccccccccc}
\mathbf{0} & 0 & 0 & \times & \times & \times & \times & \times & \times & \times & \times \\
0 & \mathbf{0} & 0 & 0 & \times & \times & \times & \times & \times & \times & \times \\
0 & 0 & \mathbf{0} & 0 & 0 & \times & \times & \times & \times & \times & \times \\
\times & 1 & 0 & \mathbf{0} & 0 & 0 & \times & \times & \times & \times & \times \\
\times & \times & 31 & 33 & \mathbf{1 0} & 52 & 68 & \times & \times & \times & \times \\
\times & \times & \times & 25 & 13 & \mathbf{7 6} & 104 & 148 & \times & \times & \times \\
\times & \times & \times & \times & 28 & 105 & \mathbf{1 2 8} & 209 & 132 & \times & \times \\
\times & \times & \times & \times & \times & 62 & 47 & \mathbf{3 6} & 22 & 6 & \times \\
\times & \times & \times & \times & \times & \times & 45 & 35 & \mathbf{1 9} & 6 & 0 \\
\times & \times & \times & \times & \times & \times & \times & 36 & 19 & \mathbf{6} & 0 \\
\times & \times & \times & \times & \times & \times & \times & \times & 19 & 0 & \mathbf{0}
\end{array}\right]
$$

In this case, the most contrail reduction solution is [llllllllllll], resulting in a contrail reduction of $176(64 \%)$ and fuel-burnt increased by $1,025 \mathrm{~kg}$. This results in a fuel consumption gradient of $5.8 \mathrm{~kg} / \mathrm{CFI}$. The most fuel-efficient solution is [1 2 $\left.\begin{array}{lllllllll}3 & 5 & 8 & 9 & 9 & 11 & 11\end{array}\right]$, with the contrail reduction of $104(38 \%)$ and fuel-burnt reduced by $2,338 \mathrm{~kg}$. This most fuel-efficient strategy achieved less contrail reduction with the benefit of less fuel consumed. efficient contrail reduction strategy using a cost function starts from the most fuel-efficient model and trades off small amount of extra fuel-burnt for large contrail reduction. The strategy would move aircraft only if the contrail reduction benefit exceeds the fuelburnt cost. The aircraft would be guided from level $l$ to $l^{\prime}$ only if

$$
\Delta C F I_{l, t}^{l^{\prime}}>\frac{\alpha}{1000} \Delta F B_{l, t}^{l^{\prime}},
$$

where $\Delta C F I_{l, t}^{l^{\prime}}$ and $\Delta F B_{l, t}^{l^{\prime}}$ are defined in Eq. (9) and Eq. (11) and $\alpha$ is the contrail reduction cost for every $1,000 \mathrm{~kg}$ fuel consumed. The fuel-efficient strategy achieves more contrail reduction for the cost of extra fuel consumption. The value of $\alpha$ has to be determined based on further understandings of the relative environmental impacts of $\mathrm{CO}_{2}$ and contrails. $\alpha$ is used in this analysis as a variable to generate different combinations of extra fuel consumption and reduction in contrails.

\section{Results}

Twenty continental U.S. enroute center contrail frequencies on August 1, 2007 were used to evaluate the contrail reducing strategy. The center contrail frequency before and after various contrail reducing strategies in twenty U.S. enroute centers at 8AM EDT is shown in Fig. 3.

In some cases, the most fuel-efficient strategy does not provide enough contrail reduction. The fuel-

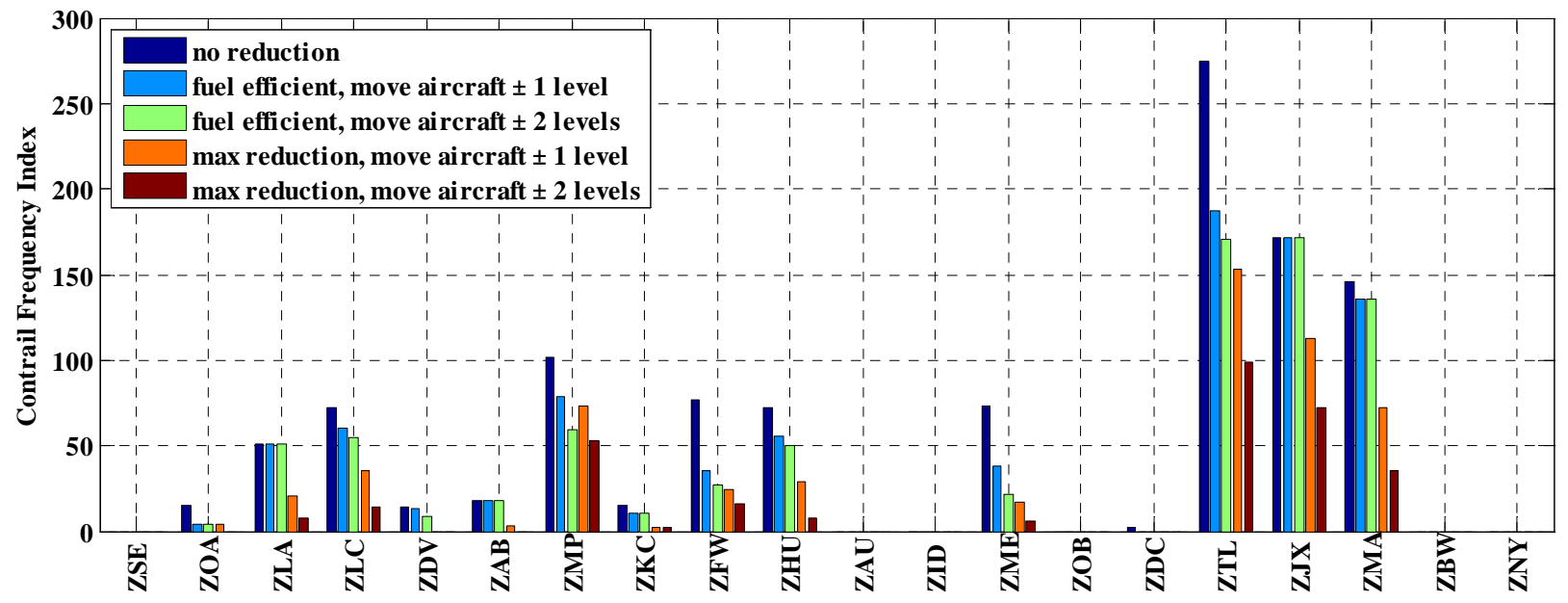

Figure 3. Center contrail frequency index before and after contrail reduction strategies at 8AM EDT on August 1, 2007. 
The strategies vary from achieving maximal contrail reduction to most fuel-efficient strategy with aircraft allowed to move one or two levels up or down. In general, allowing aircraft to move two levels provides more reduction than allowing one; maximal contrail reduction strategies provide more reduction than fuel-efficient strategies at the price of more fuel consumption. Note that fuel-efficient strategies provide no reduction at Jacksonville Center (ZJX) and Los Angeles Center (ZLA). This is because the potential contrail regions are located at the top of the centers making it impossible to fly over the regions to reduce contrail formations and save fuel. The result for the entire U.S. is summarized in Table 2. When allowing aircraft to move two levels, the maximal contrail reduction strategy reduces contrail frequency by $472(43 \%)$ more compared to the most fuelefficient strategy while using 29,237 $\mathrm{kg}(1.11 \%)$ more fuel.

Table 2. Results of contrail reduction strategies over U.S. at 8AM EDT on August 1, 2007.

\begin{tabular}{|c|c|c|}
\hline $\begin{array}{c}\text { Contrail reduction } \\
\text { strategy }\end{array}$ & $\begin{array}{c}\text { Contrail } \\
\text { reduced }\end{array}$ & Extra fuel-burnt \\
\hline Max reduction $\Delta l=1$ & $558(51 \%)$ & $5,214 \mathrm{~kg}(0.2 \%)$ \\
\hline Fuel-efficient $\Delta l=1$ & $244(23 \%)$ & $-11,134 \mathrm{~kg}(-0.42 \%)$ \\
\hline Max reduction $\Delta l=2$ & $791(72 \%)$ & $10,663 \mathrm{~kg}(0.41 \%)$ \\
\hline Fuel-efficient $\Delta l=2$ & $319(29 \%)$ & $-18574 \mathrm{~kg}(-0.71 \%)$ \\
\hline
\end{tabular}

The results of contrail reduction analysis over twenty-four-hour period on August 1, 2007 are shown in Fig. 4. The strategies allow aircraft to move two levels up or down and include the maximal contrail reduction, the most fuel-efficient, and fuelefficient strategies with contrail cost. It shows that more contrail reduction can be achieved by burning extra fuel. The results for the whole day are summarized in Table 3. On this day, the maximal reduction strategy has a $53 \%$ contrail reduction rate. The most fuel-efficient strategy has only a $8 \%$ reduction rate with $2.86 \%$ less fuel-burnt compared to maximal reduction strategy. A higher value of $\alpha$ lowers the amount of contrail reduction and uses less fuel. For $\alpha=40$, the contrail reduction is $35 \%$ with only $0.23 \%$ extra fuel consumption. Figure 5 shows the contrail reduction versus extra fuel consumption for various $\alpha$ values. This fuel-efficient strategy with contrail cost provides a flexible way to trade off between contrail reduction and fuel consumption.
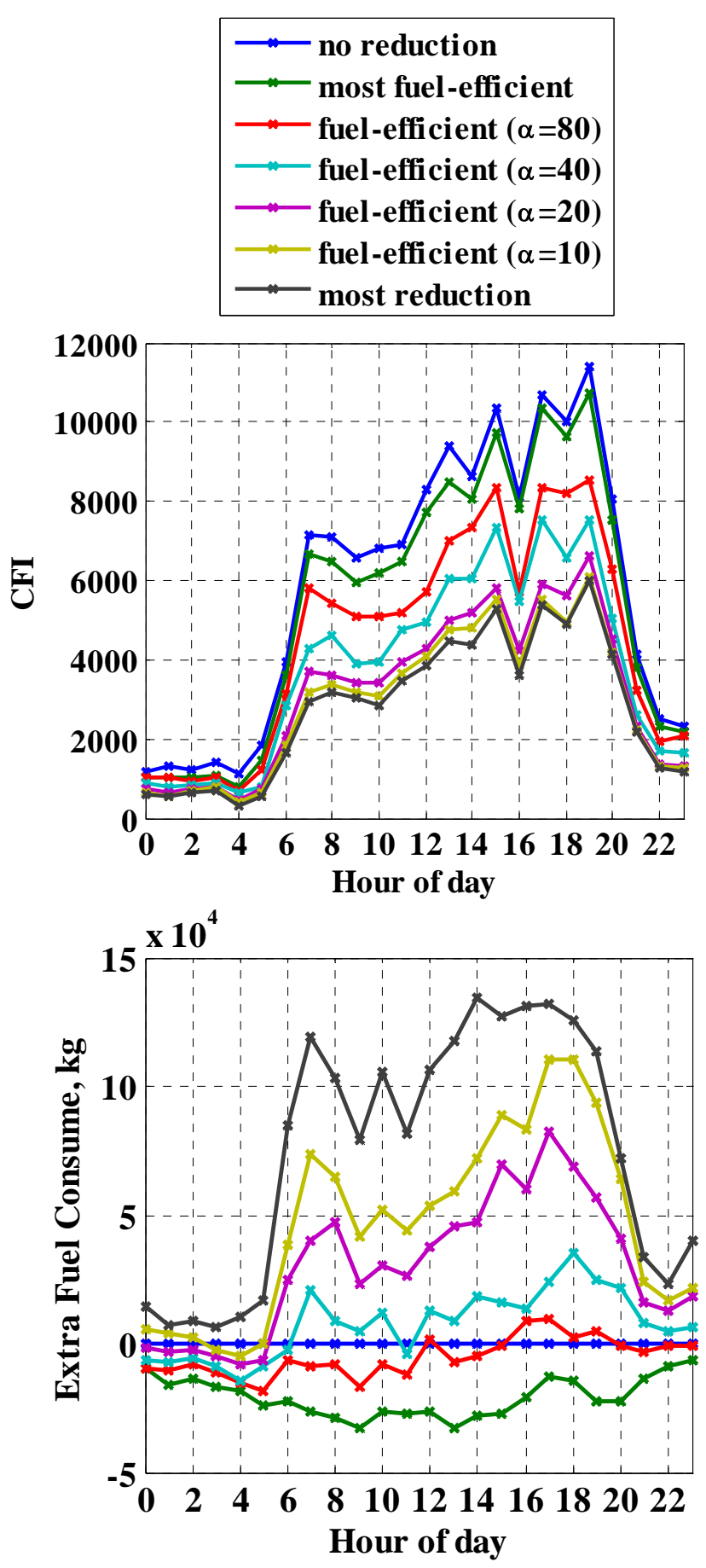

Figure 4. Contrail reduction and extra fuel-burnt using different strategies on August 1, 2007. 
Table 3. Results of contrail reduction strategies over U.S. on August 1, 2007.

\begin{tabular}{|c|c|c|}
\hline $\begin{array}{c}\text { Contrail reduction } \\
\text { strategy }\end{array}$ & $\begin{array}{c}\text { Contrail } \\
\text { reduced }\end{array}$ & Extra fuel-burnt \\
\hline Max reduction & $73,352(53 \%)$ & $1,794,924 \mathrm{~kg}(2.24 \%)$ \\
\hline Fuel-efficient $\alpha=10$ & $69,794(50 \%)$ & $1,117,341 \mathrm{~kg}(1.39 \%)$ \\
\hline Fuel-efficient $\alpha=20$ & $63,901(46 \%)$ & $720,908 \mathrm{~kg}(0.9 \%)$ \\
\hline Fuel-efficient $\alpha=40$ & $48,688(35 \%)$ & $182,567 \mathrm{~kg}(0.23 \%)$ \\
\hline Fuel-efficient $\alpha=80$ & $31,971(23 \%)$ & $-122,375 \mathrm{~kg} \mathrm{(-0.15 \% )}$ \\
\hline Most fuel-efficient & $10,260(8 \%)$ & $-496,211 \mathrm{~kg} \mathrm{(-0.62 \% )}$ \\
\hline
\end{tabular}

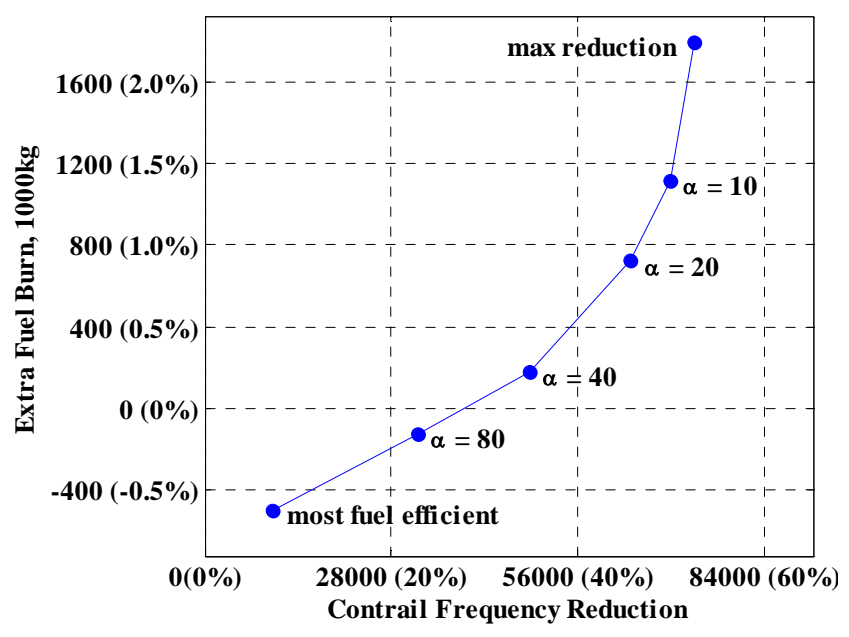

Figure 5. Contrail reduction versus extra fuelburnt on August 1, 2007.

\section{Conclusions}

The paper develops a class of strategies for reducing the persistent contrail frequency with minimal impact on extra fuel consumed and airspace congestion. The strategy of reducing the persistent contrail formations is to minimize the contrail frequency index by altering the aircraft's cruising altitude in a fuel-efficient way without adding to airspace congestion. The results show that the contrail frequency can be reduced by a significant amount without extra fuel consumption. The contrail frequency can be further reduced by using extra fuel. For the day tested, the maximal reduction strategy has a 53\% contrail reduction rate. The most fuelefficient strategy has an $8 \%$ reduction rate with $2.86 \%$ less fuel-burnt compared to maximal reduction strategy. Using a cost function which penalizes extra fuel consumed while maximizing the amount of contrail reduction provides a flexible way to trade off between contrail reduction and fuel consumption. It has a $35 \%$ contrail reduction rate with only $0.23 \%$ extra fuel consumption. The proposed fuel-efficient contrail reduction strategy provides a planning solution to reduce aviation-induced environmental impact on a daily basis.

\section{References}

[1] Jones, Mary, John Smith, 2002, Good Writing, Washington, DC, Printing Company, pp. 22-44.

[1] I. Waitz, J. Townsend, J. Cutcher-Gershenfeld, E. Greitzer, and J. Kerrebrock, "Report to the united states congress: Aviation and the environment, a national vision, framework for goals and recommended actions," Partnership for AiR Transportation Noise and Emissions Reduction, London, UK, Tech. Rep., December 2004.

[2] R. Meerkotter, U. Schumann, D. R. Doelling, P. Minnis, T. Nakajima, and Y. Tsushima, "Radiative forcing by contrails," Annales Geophysicae, vol. 17, pp. 1080-1094, 1999.

[3] S. Marquart, M. Ponater, F. Mager, and R. Sausen, "Future development of contrail cover, optical depth, and radiative forcing: Impacts of increasing air traffic and climate change," Journal of Climate, vol. 16, pp. 2890-2904, September 2003.

[4] "The environmental effects of civil aircraft in flight," Royal Commission on Environmental Pollution, London, UK, Tech. Rep., 2002.

[5] H. Mannstein and U. Schumann, "Aircraft induced contrail cirrus over europe," Meteorologische Zeitschrift, vol. 14, no. 4, pp. 549554, 2005.

[6] K. Gierens, L. Limb, and K. Eleftheratos, "A review of various strategies for contrail avoidance," The Open Atmospheric Science Journal, vol. 2, pp. $1-7,2008$.

[7] F. Noppel. and R. Singh, "Overview on contrail and cirrus cloud avoidance technology," Journal of Aircraft, vol. 44, no. 5, pp. 1721-1726, 2007.

[8] H. Mannstein, P. Spichtinger, and K. Gierens, "A note on how to avoid contrail cirrus," Transportation Research. Part D, Transport and environment, vol. 10, no. 5, pp. 421-426, September 2005. 
[9] S. E. Campbell1, N. A. Neogi, and M. B. Bragg, "An optimal strategy for persistent contrail avoidance," in AIAA Guidance, Navigation and Control Conference, ser. AIAA-2008-6515. Honolulu, HI: AIAA, August 2008.

[10] C. Fichter, S. Marquart, R. Sausen, and D. S. Lee, "The impact of cruise altitude on contrails and related radiative forcing," Meteorologische Zeitschrift, vol. 14, no. 4, pp. 563-572, August 2005.

[11] V. Williams, R. B. Noland, and R. Toumi, "Reducing the climate change impacts of aviation by restricting cruise altitudes," Transportation Research. Part D, Transport and environment, vol. 7, no. 5, pp. 451-464, November 2002.

[12] V. Williams and R. B. Noland, "Variability of contrail formation conditions and the implications for policies to reduce the climate impacts of aviation," Transportation Research. Part D, Transport and environment, vol. 10, no. 4, pp. 269-280, July 2005.

[13] P. Minnis, J. K. Ayers, M. L. Nordeen, and S. P. Weaver, "Contrail frequency over the united states from surface observations," Journal of Climate, vol. 16, no. 21, p. 34473462, November 2003.

[14] R. Palikonda, P. Minnis, D. P. Duda, and H. Mannstein, "Contrail coverage derived from 2001 avhrr data over the continental united states of america and surrounding areas," Meteorologische Zeitschrift, vol. 14, no. 4, pp. 525-536, August 2005.

[15] —, "Relating observations of contrail persistence to numerical weather analysis output," Atmospheric Chemistry and Physics, vol. 9, no. 4, pp. 1357-1364, February 2009.

[16] D. P. Duda, P. Minnis, P. K. Costulis, and R. Palikonda, "Conus contrail frequency estimated from ruc and flight track data," in European Conference on Aviation, Atmosphere, and Climate, Friedrichshafen at Lake Constance, Germany, June-July 2003.

[17] O. A. Alduchov and R. E. Eskridge, "Improved magnus form approximation of saturation vapor pressure," Journal of Applied Meteorology, vol. 35, no. 4, pp. 601-609, April 1996.

[18] N. Y. Chen, B. Sridhar, and H. K. Ng, "Prediction and use of contrail frequency index for contrail reduction strategies," in AIAA Guidance, Navigation and Control Conference. Toronto, Canada: AIAA, Aug 2010.

[19] SAGE System for assessing Aviations Global Emissions Technical Manual, 1st ed., Federal Aviation Administration, Washinton, DC, September 2005.

29th Digital Avionics Systems Conference October 3-7, 2010 